University of Texas at El Paso

ScholarWorks@UTEP

\title{
Practical Need for Algebraic (Equality-Type) Solutions of Interval Equations and for Extended-Zero Solutions
}

\author{
Ludmila Dymova \\ Czestochowa University of Technology \\ Pavel Sevastjanov \\ Czestochowa University of Technology, sevast@icis.pcz.pl \\ Andrzej Pownuk \\ The University of Texas at El Paso, ampownuk@utep.edu \\ Vladik Kreinovich \\ The University of Texas at El Paso, vladik@utep.edu
}

Follow this and additional works at: https://scholarworks.utep.edu/cs_techrep

Part of the Computer Sciences Commons, and the Mathematics Commons

Comments:

Technical Report: UTEP-CS-17-67a

To appear in Proceedings of the 12th International Conference on Parallel Processing and Applied Mathematics PPAM'17, Lublin, Poland, September 10-13, 2017

\section{Recommended Citation}

Dymova, Ludmila; Sevastjanov, Pavel; Pownuk, Andrzej; and Kreinovich, Vladik, "Practical Need for Algebraic (Equality-Type) Solutions of Interval Equations and for Extended-Zero Solutions" (2017). Departmental Technical Reports (CS). 1157.

https://scholarworks.utep.edu/cs_techrep/1157

This Article is brought to you for free and open access by the Computer Science at ScholarWorks@UTEP. It has been accepted for inclusion in Departmental Technical Reports (CS) by an authorized administrator of ScholarWorks@UTEP.For more information, please contact Iweber@utep.edu. 


\title{
Practical Need for Algebraic (Equality-Type) Solutions of Interval Equations and for Extended-Zero Solutions
}

\author{
Ludmila Dymova ${ }^{1}$, Pavel Sevastjanov ${ }^{1}$, Andrzej Pownuk ${ }^{2}$, and \\ Vladik Kreinovich ${ }^{2}$ \\ ${ }^{1}$ Institute of Computer and Infromation Science \\ Czestochowa University of Technology \\ Dabrowskiego 73, 42-200 Czestochowa, Poland \\ sevast@icis.pcz.pl \\ ${ }^{2}$ Computational Science Program, University of Texas at El Paso \\ El Paso, TX 79968, USA, ampownuk@utep.edu, vladik@utep.edu
}

\begin{abstract}
One of the main problems in interval computations is solving systems of equations under interval uncertainty. Usually, interval computation packages consider united, tolerance, and control solutions. In this paper, we explain the practical need for algebraic (equality-type) solutions, when we look for solutions for which both sides are equal. In situations when such a solution is not possible, we provide a justification for extended-zero solutions, in which we ignore intervals of the type $[-a, a]$.
\end{abstract}

\section{Practical Need for Solving Interval Systems of Equations: What Is Known}

Need for data processing. In many practical situations, we are interested in the values of quantities $y_{1}, \ldots, y_{m}$ which are difficult - or even impossible - to measure directly. For example, we can be interested in a distance to a faraway star or in tomorrow's temperature at a certain location.

Since we cannot measure these quantities directly, to estimate these quantities we must:

- find easier-to-measure quantities $x_{1}, \ldots, x_{n}$ which are related to $y_{i}$ by known formulas $y_{i}=f_{i}\left(x_{1}, \ldots, x_{n}\right)$,

- measure these quantities $x_{j}$, and

- use the results $\widetilde{x}_{j}$ of measuring the quantities $x_{j}$ to compute the estimates for $y_{i}$ :

$$
\widetilde{y}_{i}=f\left(\widetilde{x}_{1}, \ldots, \widetilde{x}_{n}\right) .
$$

Computation of these estimates is called indirect measurement or data processing.

Need for data processing under uncertainty. Measurements are never $100 \%$ accurate. Hence, the measurement result $\widetilde{x}_{j}$ is, in general, different from the 
actual (unknown) value $x_{j}$ of the corresponding quantity; in other words, the measurement errors $\Delta x_{j} \stackrel{\text { def }}{=} \widetilde{x}_{j}-x_{j}$ are, in general, different from 0 .

Because of the non-zero measurement errors, the estimates $\widetilde{y}_{i}$ are, in general, different from the desired values $y_{i}$. It is therefore desirable to know how accurate are the resulting estimates.

Need for interval uncertainty and interval computations. The manufacturer of the measuring instrument usually provides us with an upper bound $\Delta_{j}$ on the measurement error: $\left|\Delta x_{j}\right| \leq \Delta_{j}$; see, e.g., [8]. If no such upper bound is known, i.e., if the reading of the instrument can be as far away from the actual value as possible, then this is not a measuring instrument, this is a wild-guessgenerator.

Sometimes, we also know the probabilities of different values $\Delta x_{j}$ within this interval; see, e.g., $[8,15]$. However, in many practical situations, the upper bound is the only information that we have [8]. In this case, after we know the result $\widetilde{x}_{j}$ of measuring $x_{j}$, the only information that we have about the actual (unknown) value $x_{j}$ is that this value belongs to the interval $\left[\underline{x}_{j}, \bar{x}_{j}\right]$, where $\underline{x}_{j} \stackrel{\text { def }}{=} \widetilde{x}_{j}-\Delta_{j}$ and $\bar{x}_{j} \stackrel{\text { def }}{=} \widetilde{x}_{j}+\Delta_{j}$.

In this case, the only thing that we can say about each value $y_{i}=$ $f_{i}\left(x_{1}, \ldots, x_{n}\right)$ is that this value belongs to the range

$$
\left\{f_{i}\left(x_{1}, \ldots, x_{n}\right): x_{1} \in\left[\underline{x}_{1}, \bar{x}_{1}\right], \ldots, x_{n} \in\left[\underline{x}_{n}, \bar{x}_{n}\right]\right\} .
$$

Computation of this range is one of the main problems of interval computations; see, e.g., $[3,6]$.

Sometimes, we do not know the exact dependence. The above text described an ideal case, when we know the exact dependence $y_{i}=f_{i}\left(x_{1}, \ldots, x_{n}\right)$ between the desired quantities $y_{i}$ and the easier-to-measure quantities $x_{j}$. In practice, often, we do not know the exact dependence. Instead, we know that the dependence belongs to a finite-parametric family of dependencies, i.e., that

$$
y_{i}=f_{i}\left(x_{1}, \ldots, x_{n}, a_{1}, \ldots, a_{k}\right)
$$

for some parameters $a_{1}, \ldots, a_{k}$.

For example, we may know that $y_{i}$ is a linear function of the quantities $x_{j}$, i.e., that $y_{i}=c_{i}+\sum_{j=1}^{n} c_{i j} \cdot x_{j}$ for some coefficients $c_{i}$ and $c_{i j}$.

The presence of these parameters complicates the corresponding data processing problem. Depending on what we know about the parameters, we have different situations.

Simplest situation, when we know the exact values of all the parameters. The simplest situation is when we know the exact values of these parameters. In this case, the dependence of $y_{i}$ on $x_{j}$ is known, and we have the same problem of computing the range as before.

Specific case: control solution. Sometimes, not only we know the values $a_{\ell}$ of these parameters, but we can also control these values, by setting them to any 
values within certain intervals $\left[\underline{a}_{\ell}, \bar{a}_{\ell}\right]$. By setting the appropriate values of the parameters, we can change the values $y_{i}$. This possibility naturally leads to the following problem:

- we would like the values $y_{i}$ to be within some given ranges $\left[\underline{y}_{i}, \bar{y}_{i}\right]$; for example, we would like the temperature to be within a comfort zone;

- in this case, it is desirable to find the range of possible values of $x_{j}$ for which, by applying appropriate controls $a_{i} \in\left[\underline{a}_{\ell}, \bar{a}_{\ell}\right]$, we can place the values $y_{i}$ within these intervals.

In the degenerate case, when all the intervals for $y_{i}$ and $a_{\ell}$ are just points, this means solving the system of equations $y=f(x, a)$, where we denoted $y \stackrel{\text { def }}{=}$ $\left(y_{1}, \ldots, y_{m}\right), x \stackrel{\text { def }}{=}\left(x_{1}, \ldots, x_{n}\right)$, and $a \stackrel{\text { def }}{=}\left(a_{1}, \ldots, a_{k}\right)$. From this viewpoint, the above problem can be viewed as an interval generalization of the problem of solving a system of equations, or, informally, as a problem of solving the corresponding interval system of equations.

The set $X$ of all appropriate values $x=\left(x_{1}, \ldots, x_{n}\right)$ can be formally described as

$$
X=\left\{x: \text { for some } a_{\ell} \in\left[\underline{a}_{\ell}, \bar{a}_{\ell}\right], f_{i}\left(x_{1}, \ldots, x_{n}, a_{1}, \ldots, a_{k}\right) \in\left[\underline{y}_{i}, \bar{y}_{i}\right] \text { for all } i\right\} .
$$

This set is known as the control solution to the corresponding interval system of equations $[3,14]$.

Situation when we need to find the parameters from the data. Sometimes, we know that the values $a_{i}$ are the same for all the cases, but we do not know these values. These values must then be determined based on measurements: we measure $x_{j}$ and $y_{i}$ several times, and we find the values of the parameters $a_{\ell}$ that match all the measurement results.

Let us number all membership cycles by values $c=1, \ldots, C$. After each cycle of measurements, we conclude that:

- the actual (unknown) value of $x_{j}^{(c)}$ is in the interval $\left[\underline{x}_{j}^{(c)}, \bar{x}_{j}^{(c)}\right]$ and

- the actual value of $y_{i}^{(c)}$ is in the interval $\left[\underline{y}_{i}^{(c)}, \bar{y}_{i}^{(c)}\right]$.

We want to find the set $A$ of all the values $a$ for which $y^{(c)}=f\left(x^{(c)}, a\right)$ for some $x^{(c)}$ and $y^{(c)}$ :

$$
A=\left\{a: \forall c \exists x_{j}^{(c)} \in\left[\underline{x}_{j}^{(c)}, \bar{x}_{j}^{(c)}\right] \exists y_{i}^{(c)} \in\left[\underline{y}_{i}^{(c)}, \bar{y}_{i}^{(c)}\right]\left(f\left(x^{(c)}, a\right)=y^{(c)}\right)\right\} .
$$

This set $A$ is known as the united solution to the interval system of equations [3, 14].

Comment. To avoid confusion, it is worth mentioning that our notations are somewhat different from the notations used in $[3,14]$.

The main reason for this difference is that the main focus of this paper is on the motivations for different types of solutions. As a result, we use the notations related to the meaning of the corresponding variables. In general, in 
our description, $y$ denotes the desired (difficult-to-measure) quantities, $x$ denote easier-to-measure quantities, and $a$ denote parameters of the dependence between these quantities.

Within this general situation, we can have different problems.

- In some cases, we have some information about the parameters $a$, and we need to know the values $x$ - this is the case of the control solution.

- In other practical situations, we have some information about the quantities $x$, and we need to know the values $a$ - this is the case, e.g., for the united solution.

As a result, when we use our meaning-of-variables notations, sometimes $x$ 's are the unknowns, and sometimes $a$ 's are the unknowns.

Alternatively, if we were interested in actually solving the corresponding problems, it would be more appropriate to use different notations, in which, e.g., the unknown is always denoted by $x$ and the known values are denoted by $a$-irrespective of the physical meaning of the corresponding variables. In these notations, the united solution would take a different form

$$
X=\left\{x: \forall c \exists a_{j}^{(c)} \in\left[\underline{a}_{j}^{(c)}, \bar{a}_{j}^{(c)}\right] \exists y_{i}^{(c)} \in\left[\underline{y}_{i}^{(c)}, \bar{y}_{i}^{(c)}\right]\left(f\left(x, a^{(c)}\right)=y^{(c)}\right)\right\} .
$$

What can we do once we have found the range of possible values of $a$. Once we have found the set $A$ of possible values of $a$, we can first find the range of possible values of $y_{i}$ based on the measurement results, i.e., find the range

$$
\left\{f_{i}\left(x_{1}, \ldots, x_{n}, a\right): x_{j} \in\left[\underline{x}_{j}, \bar{x}_{j}\right] \text { and } a \in A\right\} .
$$

This is a particular case of the main problem of interval computations.

If we want to make sure that each value $y_{i}$ lies within the given bounds $\left[\underline{y}_{i}, \bar{y}_{i}\right]$, then we must find the set $X$ of possible values of $x$ for which $f_{i}(x, a)$ is within these bounds for all possible values $a \in A$, i.e., the set

$$
X=\left\{x: \forall a \in A \forall i\left(f_{i}(x, a) \in\left[\underline{y}_{i}, \bar{y}_{i}\right]\right)\right\} .
$$

This set is known as the tolerance solution to the interval system of equations [3, $14]$.

Sometimes, we know that the values $a$ may change. In the previous text, we consider the situations when the values $a_{\ell}$ are either fixed forever, or can be changed by us. In practice, these values may change in an unpredictable way e.g., if these parameters represent some physical processes that influence $y_{i}$ 's. We therefore do not know the exact values of these parameters, but what we do know is some a priori bounds on these values.

We may know bounds $\left[\underline{a}_{\ell}, \bar{a}_{\ell}\right]$ on each parameter, in which case the set $A$ of all possible combinations $a=\left(a_{1}, \ldots, a_{k}\right)$ is simply a box:

$$
A=\left[\underline{a}_{1}, \bar{a}_{1}\right] \times \ldots \times\left[\underline{a}_{k}, \bar{a}_{k}\right] .
$$


We may also have more general sets $A$ - e.g., ellipsoids.

In this case, we can still solve the same two problems whose solutions we described above; namely:

- we can solve the main problem of interval computations - the problem of computing the range - and find the set $Y$ of possible values of $y$;

- we can also solve the corresponding tolerance problem and find the set of values $x$ that guarantee that each $y_{i}$ is within the desired interval.

Is this all there is? There are also more complex problems (see, e.g., [14]), but, in a nutshell, most practical problems are either range estimation, or finding control, united, or tolerance solution. These are the problems solved by most interval computation packages $[3,6]$.

Is there anything else? In this paper, we show that there is an important class of practical problems that does not fit into one of the above categories. To solve these practical problems, we need to use a different notion of a solution to interval systems of equations: the notion of an algebraic (equality-type) solution, the notion that has been previously proposed and theoretically analyzed $[1,2,5$, $7,9,12-14]$ but is not usually included in interval computations packages.

\section{Remaining Problem of How to Find the Set $A$ Naturally Leads to Algebraic (Equality-Type) Solutions to Interval System of Equations}

Finding the set $A$ : formulation of the problem. In the previous text, we assumed that when the values of the parameter $a$ can change, we know the set $A$ of possible values of the corresponding parameter vector. But how do we find this set?

What information we can use to find the set $A$. All the information about the real world comes from measurements - either directly from measurements, or by processing measurement results. The only relation between the parameters $a$ and measurable quantities is the formula $y=f(x, a)$. Thus, to find the set $A$ of possible values of $a$, we need to use measurements of $x$ and $y$.

We can measure both $x$ and $y$ many times. As a result, we get:

- the set $X$ of possible values of the vector $x$ and

- the set $Y$ of possible values of the vector $y$.

Both sets can be boxes, or they can be more general sets.

Based on these two sets $X$ and $Y$, we need to find the set $A$.

In this problem, it is reasonable to assume that $x$ and $a$ are independent in some reasonable sense. Let us formulate this requirement in precise terms.

Independence: towards a formal definition. The notion of independence is well known in the probabilistic case, where it means that probability of getting a value $x \in X$ does not depend on the value $a \in A: P(x \mid a)=P\left(x \mid a^{\prime}\right)$ for all 
$a, a^{\prime} \in A$. An interesting corollary of this definition is that, in spite of being formulated in a way that is asymmetric with respect to $x$ and $a$, this definition is actually symmetric: one can prove that $a$ is independent of $x$ if and only if $x$ is independent of $a$.

In the interval case, we do not know the probabilities, we only know which pairs $(x, a)$ are possible and which are not. In other words, we have a set $S \subseteq$ $X \times A$ of possible pairs $(x, a)$. It is natural to say that the values $x$ and $a$ are independent if the set of possible values of $x$ does not depend on $a$. Thus, we arrive at the following definition.

Definition 1. Let $S \subseteq X \times A$ be a set.

- We say that a pair $(x, a)$ is possible if $(x, a) \in S$.

- Let $x \in X$ and $a \in A$. We say that a value $x$ is possible under $a$ if $(x, a) \in S$. The set of possible-under-a values will be denoted by $S_{a}$.

- We say that the variables $x$ and $a$ are independent if $S_{a}=S_{a^{\prime}}$ for all $a, a^{\prime}$ from the set $A$.

Proposition 1. Variable $x$ and $a$ are independent if and only if $S$ is a Cartesian product, i.e., $S=s_{x} \times s_{a}$ for some $s_{x} \subseteq X$ and $s_{a} \subseteq A$.

Proof. If $S=s_{x} \times s_{a}$, then $S_{a}=s_{x}$ for each $a$ and thus, $S_{a}=S_{a^{\prime}}$ for all $a, a^{\prime} \in A$.

Vice versa, let us assume that $x$ and $a$ are independent. Let us denote the common set $S_{a}=S_{a^{\prime}}$ by $s_{x}$. Let us denote by $s_{a}$, the set of all possible values $a \in A$, i.e., the set of all $a \in A$ for which $(x, a) \in S$ for some $x \in X$. Let us prove that in this case, $S=s_{x} \times s_{a}$.

Indeed, if $(x, a) \in S$, then, by definition of the set $s_{x}$, we have $x \in S_{a}=s_{x}$, and, by definition of the set $s_{a}$, we have $a \in s_{a}$. Thus, by the definition of the Cartesian product $B \times C$ as the set of all pairs $(b, c)$ of all pairs of elements $b \in B$ and $c \in C$, we have $(x, a) \in s_{x} \times s_{a}$.

Vice versa, let $(x, a) \in s_{x} \times s_{a}$, i.e., let $x \in s_{x}$ and $a \in s_{a}$. By definition of the set $s_{x}$, we have $S_{a}=s_{x}$, thus $x \in S_{a}$. By definition of the set $S_{a}$, this means that $(x, a) \in S$. The proposition is proven.

As a corollary, we can conclude that the independence relation is symmetric similarly to the probabilistic case.

Corollary. Variables $x$ and $a$ are independent if and only if $a$ and $x$ are independent.

Proof: indeed, both case are equivalent to the condition that the set $S$ is a Cartesian product.

What can we now conclude about the dependence between $A, X$, and $Y$. Since we assumed that $a$ and $x$ are independent, we can conclude that the set of possible values of the pair $(x, a)$ is the Cartesian product $X \times A$. For each such pair, the value of $y$ is equal to $y=f(x, a)$. Thus, the set $Y$ is equal to the range of $f(x, a)$ when $x \in X$ and $a \in A$. 
The resulting solutions to interval systems of equations. So, we look for sets $A$ for which

$$
Y=f(X, A) \stackrel{\text { def }}{=}\{f(x, a): x \in X \text { and } a \in A\} .
$$

It is reasonable call the set $A$ satisfying this property an equality-type solution to the interval system of equations.

Such solutions for the interval system of equations $y=f(x, a)$, in which we want the interval versions $Y$ and $f(X, A)$ of both sides of the equation to be exactly equal, are known as algebraic or, alternatively, formal solutions; see, e.g., $[1,2,5,7,9,12-14]$.

\section{What If the Interval System of Equations Does Not Have an Algebraic (Equality-Type) Solution: A Justification for Enhanced-Zero Solutions}

But what if an equality-type solution is impossible: analysis of the problem. The description in the previous section seems to make sense, but sometimes, the corresponding problem has no solutions. For example, in the simplest case when $m=n=k=1$ and $f(x, a)=x+a$, if we have $Y=[-1,1]$ and $X=[-2,2]$, then clearly the corresponding equation $Y=X+A$ does not have a solution: no matter what set $A$ we take the width of the resulting interval $X+A$ is always larger than or equal to the width $w(X)=4$ of the interval $X$ and thus, cannot be equal to $w(Y)=2$. What shall we do in this case? How can we then find the desired set $A$ ?

Of course, this would not happen if we had the actual ranges $X$ and $Y$, but in reality, we only have estimates for these ranges. So, the fact that we cannot find $A$ means something is wrong with these estimates.

How are ranges $X$ and $Y$ estimated in the first place? To find out what can be wrong, let us recall how the ranges can be obtained from the experiments. For example, in the 1-D case, we perform several measurements of the quantity $x_{1}$ in different situations. Based on the corresponding measurement results $x_{1}^{(c)}$, we conclude that the interval of possible values must include the set $\left[\underline{x}_{1}, \bar{x}_{1} \widetilde{]}\right.$, where $\underline{x} \approx \stackrel{\text { def }}{=} \min _{c} x_{1}^{(c)}$ and $\bar{x} \approx \stackrel{\text { def }}{=} \max _{c} x_{1}^{(c)}$. Of course, we can also have some values outside this interval - e.g., for a uniform distribution on an interval $[0,1]$, the interval formed by the smallest and the largest of the $C$ random numbers is slightly narrower than $[0,1]$; the fewer measurement we take, the narrower this interval.

So, to estimate the actual range, we inflate the interval $\left[\underline{x}_{1}^{\approx}, \bar{x}_{1}^{\approx}\right]$. In these terms, the fact that we have a mismatch between $X$ and $Y$ means that one of these intervals was not inflated enough.

The values $x$ correspond to easier-to-measure quantities, for which we can make a large number of measurements and thus, even without inflation, get pretty accurate estimates of the actual range $X$. On the other hand, the values 
$y$ are difficult to measure; for these values, we do not have as many measurement results and thus, there is a need for inflation.

From this viewpoint, we can safely assume that the range for $X$ is reasonably accurate, but the range of $Y$ needs inflation.

So how do we find $A$ ? In view of the above analysis, if there is no set $A$ for which $Y=f(X, A)$, the proper solution is to inflate each components of the set $Y$ so that the system becomes solvable.

To make this idea precise, let us formalize what is an inflation.

What is an inflation: analysis of the problem. We want to define a mapping $I$ that transforms each non-degenerate interval $\mathrm{x}=[\underline{x}, \bar{x}]$ into a wider interval

$$
I(\mathbf{x}) \supset \mathbf{x} .
$$

What are the natural properties of this transformation? The numerical value $x$ of the corresponding quantity depends on the choice of the measuring unit, on the choice of the starting point, and - sometimes - on the choice of direction.

- For example, we can measure temperature $t_{C}$ in Celsius, but we can also use a different measuring unit and a different starting point and get temperatures in Fahrenheit $t_{F}=1.8 \cdot t_{C}+32$.

- We can use the usual convention and consider the usual signs of the electric charge, but we could also use the opposite signs - then an electron would be a positive electric charge.

It is reasonable to require that the result of the inflation transformation does not change if we simply change the measuring units or change the starting point or change the sign:

- Changing the starting point leads to a new interval $[\underline{x}, \bar{x}]+x_{0}=\left[\underline{x}+x_{0}, \bar{x}+x_{0}\right]$ for some $x_{0}$.

- Changing the measuring unit leads to $\lambda \cdot[\underline{x}, \bar{x}]=[\lambda \cdot \underline{x}, \bar{x}]$ for some $\lambda>0$.

- Changing the sign leads to $-[\underline{x}, \bar{x}]=[-\bar{x},-\underline{x}]$.

Thus, we arrive at the following definition.

Definition 2. By an inflation operation (or just inflation, for short) we mean a mapping that maps each non-degenerate interval $\mathbf{x}=[\underline{x}, \bar{x}]$ into a wider interval $I(\mathbf{x}) \supset \mathbf{x}$ so that:

- for every $x_{0}$, we have $I\left(\mathbf{x}+x_{0}\right)=I(\mathbf{x})+x_{0}$;

- for every $\lambda>0$, we have $I(\lambda \cdot \mathbf{x})=\lambda \cdot I(\mathbf{x})$; and

- we have $I(-\mathbf{x})=-I(\mathbf{x})$.

Proposition 2. Every inflation operation has the form

$$
[\widetilde{x}-\Delta, \widetilde{x}+\Delta] \rightarrow[\widetilde{x}-\alpha \cdot \Delta, \widetilde{x}+\alpha \cdot \Delta]
$$

for some $\alpha>1$. 
Comment. A similar result was proven in [4].

Proof. It is easy to see that the above operation satisfies all the properties of an inflation. Let us prove that, vice versa, every inflation has this form.

Indeed, for intervals $\mathbf{x}$ of type $[-\Delta, \Delta]$, we have $-\mathbf{x}=\mathbf{x}$, thus $I(\mathbf{x})=I(-\mathbf{x})$. On the other hand, due to the third property of an inflation, we should have $I(-\mathbf{x})=-I(\mathbf{x})$. Thus, for the interval $[\underline{v}, \bar{v}] \stackrel{\text { def }}{=} I(\mathbf{x})$, we should have $-[\underline{v}, \bar{v}]=$ $[-\bar{v},-\underline{v}]=[\underline{v}, \bar{v}]$ and thus, $\underline{v}=-\bar{v}$. So, we have $I([-\Delta, \Delta])=\left[-\Delta^{\prime}(\Delta), \Delta^{\prime}(\Delta)\right]$ for some $\Delta^{\prime}$ depending on $\Delta$. Since we should have $[-\Delta, \Delta] \subset I([-\Delta, \Delta])$, we must have

$$
\Delta^{\prime}(\Delta)>\Delta
$$

Let us denote $\Delta^{\prime}(1)$ by $\alpha$. Then, $\alpha>1$ and $I([-1,1])=[-\alpha, \alpha]$. By applying the second property of the inflation, with $\lambda=\Delta$, we can then conclude that $I([-\Delta, \Delta])=[-\alpha \cdot \Delta, \alpha \cdot \Delta]$. By applying the first property of the inflation operation, with $x_{0}=\widetilde{x}$, we get the desired equality

$$
I([\widetilde{x}-\Delta, \widetilde{x}+\Delta])=[\widetilde{x}-\alpha \cdot \Delta, \widetilde{x}+\alpha \cdot \Delta] .
$$

The proposition is proven.

So how do we find $A$ ? We want to make sure that $f(X, A)$ is equal to the result of a proper inflation of $Y$.

How can we tell that an interval $Y^{\prime}$ is the result of a proper inflation of $Y$ ? One can check that this is equivalent to the fact that the difference $Y^{\prime}-Y$ is a symmetric interval containing 0 ; such intervals are known as extended zeros $[10$, $11]$.

Thus, if we cannot find the set $A$ for which $Y=f(X, A)$, we should look for the set $A$ for which the difference $f(X, A)-Y$ is an extended zero.

Historical comment. This idea was first described in $[10,11]$; in this paper, we provide a new theoretical justification of this idea.

Multi-D case. What if we have several variables, i.e., $m>1$ ? In this case, we may have different inflations for different components $Y_{i}$ of the set $Y$, so we should look for the set $A$ for which, for all $i$, the corresponding difference $f_{i}(X, A)-Y_{i}$ is an extended zero.

\section{Acknowledgments}

This work was supported in part by the grant DEC-2013/11/B/ST6/00960 from the National Science Center (Poland), by the US National Science Foundation grants HRD-0734825 and HRD-1242122 (Cyber-ShARE Center of Excellence) and DUE-0926721, and by an award "UTEP and Prudential Actuarial Science Academy and Pipeline Initiative" from Prudential Foundation.

The authors are thankful to the anonymous referees for valuable suggestions. 


\section{References}

1. S. Chakraverty, M. Hladík, and D. Behera, "Formal solution of an interval system of linear equations with an application in static responses of structures with interval forces", Applied Mathematical Modeling, 2017, Vol. 50, pp. 105-117.

2. S. Chakraverty, M. Hladík, and N. R. Mahato, "A sign function approach to solve algebraically interval system of linear equations for nonnegative solutions", Fundamenta Informaticae, 2017, Vol. 152, pp. 13-31.

3. L. Jaulin, M. Kiefer, O. Dicrit, and E. Walter, Applied Interval Analysis, Springer, London, 2001.

4. V. Kreinovich, S. A. Starks, and G. Mayer, "On a Theoretical Justification of The Choice of Epsilon-Inflation in PASCAL-XSC", Reliable Computing, 1997, Vol. 3, No. 4, pp. 437-452.

5. A. Lakeyev, "On the computational complexity of the solution of linear systems with moduli", Reliable Computing, 1996, Vol. 2, No. 2, pp. 125-131.

6. R. E. Moore, R. B. Kearfott, and M. J. Cloud, Introduction to Interval Analysis, SIAM, Philadelphia, 2009.

7. K. Nickel, "Die Auflösbarkeit linearer Kreisscheineb- und IntervallGleichingssyteme", Linear Algebra and its Applications, 1982, Vol. 44, pp. 19-40.

8. S. G. Rabinovich, Measurement Errors and Uncertainty: Theory and Practice, Springer Verlag, Berlin, 2005.

9. K. Ratschek and W. Sauer, "Linear interval equations", Computing, 1982, Vol. 25, pp. 105-115.

10. P. Sevastjanov and L. Dymova, "Fuzzy solution of interval linear equations", Proceedings of the 7th International Conference on Parallel Processing and Applied Mathematics PPAM'2017, Gdansk, Poland, 2007, pp. 1392-1399.

11. P. Sevastjanov and L. Dymova, "A new method for solving interval and fuzzy equations: linear case", Information Sciences, 2009, Vol. 17, pp. 925-937.

12. S. P. Shary, "Algebraic approach to the interval linear static identification, tolerance, and control problems, or one more application of Kaucher arithmetic", Reliable Computing, 1996, Vol. 2, No. 1, pp. 3-33.

13. S. P. Shary, "Algebraic approach in the 'outer problem' for interval linear equations", Reliable Computing, 1997, Vol. 3, No. 2, pp. 103-135.

14. S. P. Shary, "A new technique in systems analysis under interval uncertainty and ambiguity", Reliable Computing, 2002, Vol. 8, pp. 321-418.

15. D. J. Sheskin, Handbook of Parametric and Nonparametric Statistical Procedures, Chapman and Hall/CRC, Boca Raton, Florida, 2011. 